Musées, Patrimoine et Culture scientifiques et techniques

$176 \mid 2018$

mars-avril 2018

\title{
Nouveauté dans les musées et lieux d'exposition: les apprentis en muséographie
}

Serge Chaumier

\section{OpenEdition}

\section{Journals}

Édition électronique

URL : http://journals.openedition.org/ocim/2017

DOI : 10.4000/ocim.2017

ISSN : 2108-646X

Éditeur

OCIM

Édition imprimée

Date de publication : 1 mars 2018

Pagination : 12-16

ISSN : 0994-1908

Référence électronique

Serge Chaumier, "Nouveauté dans les musées et lieux d'exposition : les apprentis en muséographie », La Lettre de I'OCIM [En ligne], 176 | 2018, mis en ligne le 01 mars 2019, consulté le 10 octobre 2020. URL : http://journals.openedition.org/ocim/2017 ; DOI : https://doi.org/10.4000/ocim.2017

Ce document a été généré automatiquement le 10 octobre 2020

Tous droits réservés 


\title{
Nouveauté dans les musées et lieux d'exposition : les apprentis en muséographie
}

\author{
Serge Chaumier
}

Les apprenties du master Muséographie Expographie de l'université d'Artois sont dans les musées municipaux, les conservations départementales, les établissements publics, les entreprises ou les agences. Des structures diverses de culture scientifique, comme des musées historiques,

ethnographique ou de société, des musées d'arts, des centres d'arts contemporains comme ici à Rouen au SHED à l'occasion de l'exposition Ann Veronica Janssens.

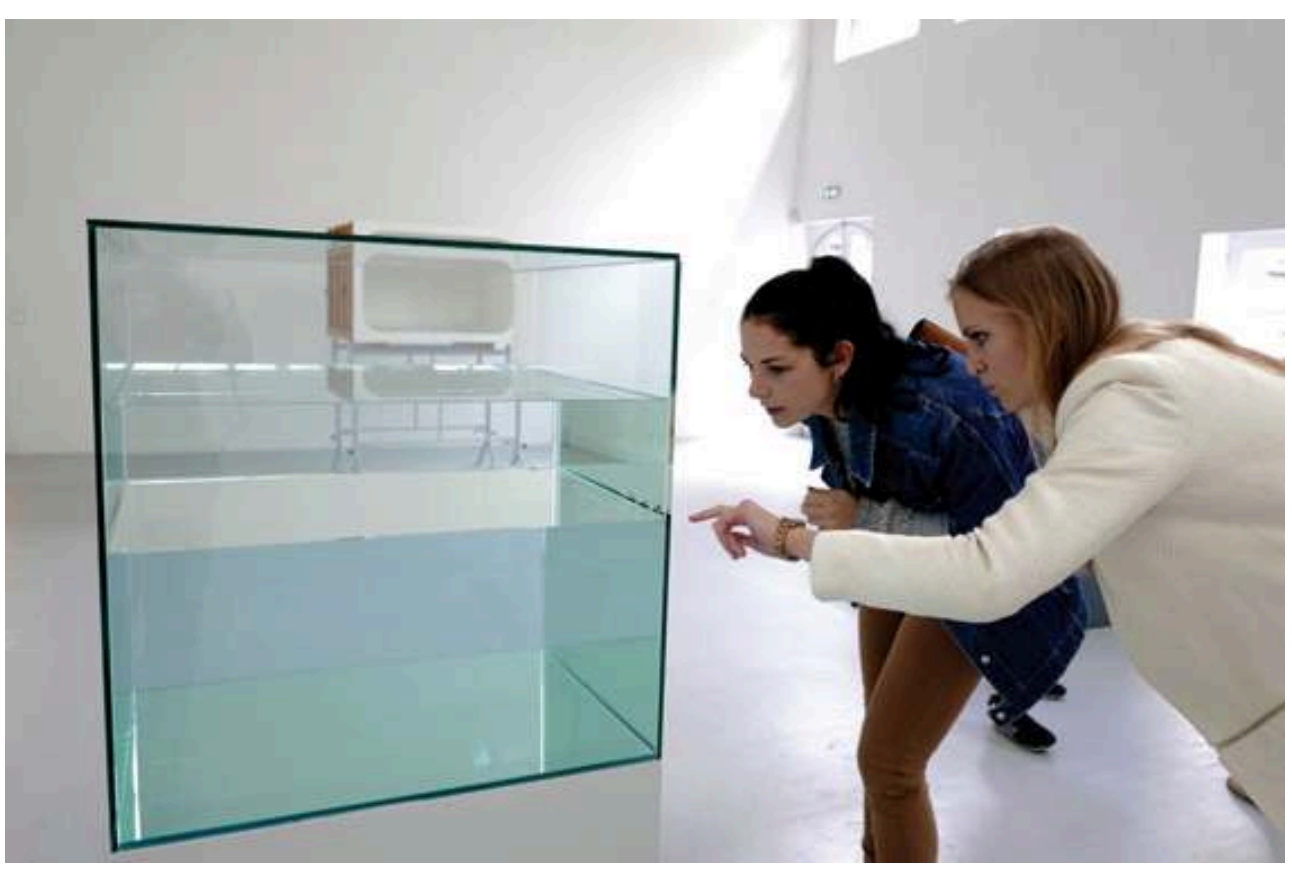

(c) Art de Muser

Si l'apprentissage était jusque-là plutôt connu dans les filières courtes et manuelles, le dispositif s'étend depuis une quinzaine d'années aux filières du supérieur. Si les 
secteurs de la banque, de l'informatique ou encore des techniques, recrutent désormais massivement des commerciaux, des programmistes ou des ingénieurs, le milieu culturel et plus spécifiquement de l'exposition ne disposait pas, jusqu'à peu, de formation universitaire dispensée en apprentissage. Quelques formations de niveau master existent le plus souvent dans le milieu du tourisme et du patrimoine et désormais pour les métiers de la conception d'exposition, de la conservation et régie, de la médiation ou de l'administration de projets culturels. Quels sont les avantages et les caractéristiques de cette démarche?

2 L'esprit de l'apprentissage réside dans le compagnonnage. C'est vrai entre l'apprenti et le formateur mais aussi avec son maître d'apprentissage. Ces deux référents, l'un dans le centre de formation et l'autre dans l'entreprise, accompagnent l'apprenti dans sa professionnalisation pour lui transmettre des savoir-faire, mais aussi des clés pour entrer dans la carrière. Il s'agit d'abord de lui permettre de comprendre l'entreprise, de l'accompagner dans la maîtrise des codes et in fine de lui permettre de prendre confiance en lui pour assumer un rôle dans l'organigramme. L'objectif est que l'apprenti soit très vite autonome dans son poste, devienne un collègue à part entière, responsable d'une mission bien définie.

\section{La spécificité de l'apprentissage : former des salariés}

Pour aborder l'apprentissage, il faut l'envisager à l'inverse des stages, dispositif mieux connu des institutions. Il ne s'agit pas ici d'un étudiant qui vient en entreprise pour en découvrir les coulisses et y assumer une mission mais d'un salarié qui va se former lors de session de regroupement dans un Centre de Formation pour Apprentis (CFA). Ceci veut dire qu'un apprenti est d'abord un jeune salarié : il en a les obligations, les devoirs et les droits et qu'il est pour un temps long dans l'entreprise, en général pour une ou deux années. Sur une année, le temps de formation est d'environ trois mois et en entreprise d'environ 9 mois, congés inclus. Si les trois premiers mois sont un temps d'acclimatation et de prise de connaissance des fonctionnements internes ainsi que d'appropriation des missions confiées, une montée en puissance au cours de l'année conduit pour finir à ce que l'apprenti soit un salarié comme un autre, le jeune professionnel est devenu collègue.

\section{L'avantage pour l'apprenti}

4 C'est une opportunité pour un jeune professionnel de pouvoir faire ses preuves et de disposer d'une année d'exercice comme salarié dans une structure, plutôt que de 3 mois de stage comme il est courant dans les formations de master. Bien que disposant d'un salaire somme toute modeste, c'est malgré tout une vraie expérience de salarié qui le place dans une situation de responsabilité. Selon les régions, l'âge de l'apprenti doit être de moins de 27 ou 30 ans (davantage pour un salarié au statut handicapé ou dans le cadre de la création d'entreprise). Des aides à la mobilité, à l'hébergement et restauration, à l'équipement de l'apprenti, et parfois à l'entreprise qui recrute existent, variables selon les régions. 


\section{L'encadrement et les relations partenariales}

5 Chaque apprenti dispose d'un double encadrement : un maitre de formation en entreprise et un tuteur au CFA, qui sont ses référents. Contrairement au stage classique, où l'étudiant est généralement peu encadré par le référent académique, le référent du CFA suit étroitement l'évolution de l'apprenti en entreprise, avec deux ou trois visites sur site dans l'année, une au moins à l'installation et une en fin d'apprentissage, et fait le point régulièrement à chaque retour au CFA.

6 Les missions sont suivies et le CFA peut apporter des compétences nécessaires, des ressources, des contacts. Les référents en entreprise et au CFA communiquent régulièrement entre eux, notamment en Hauts-de-France au travers de la plateforme partagée en ligne MySup mise en place par Formasup, l'organisme gestionnaire de l'apprentissage. Un livret dématérialisé, des fiches de communication, de suivi et d'évaluation sont ainsi partagés et validés par chacune des parties prenantes. Le suivi permet ainsi de construire un portfolio de compétences du jeune salarié.

Un tournage au musée des Beaux-Arts d'Arras. Plusieurs formes d'initiation aux dispositifs de médiation et d'actions culturelles sont conduites par des apprenties, tant durant la Biennale d'art contemporain Appel d'Air, que pour le concours du Sitem Musée(em)portables, ou durant les expositions réalisées en partenariat : par exemple avec des migrants à La Manufacture des Flandres en 2017. Des reportages sur les médiations nourrissent chaque année une plateforme d'observations des médiations, accompagnant par ailleurs un travail d'écriture de critiques publiées sur le site de L'Art de Muser.

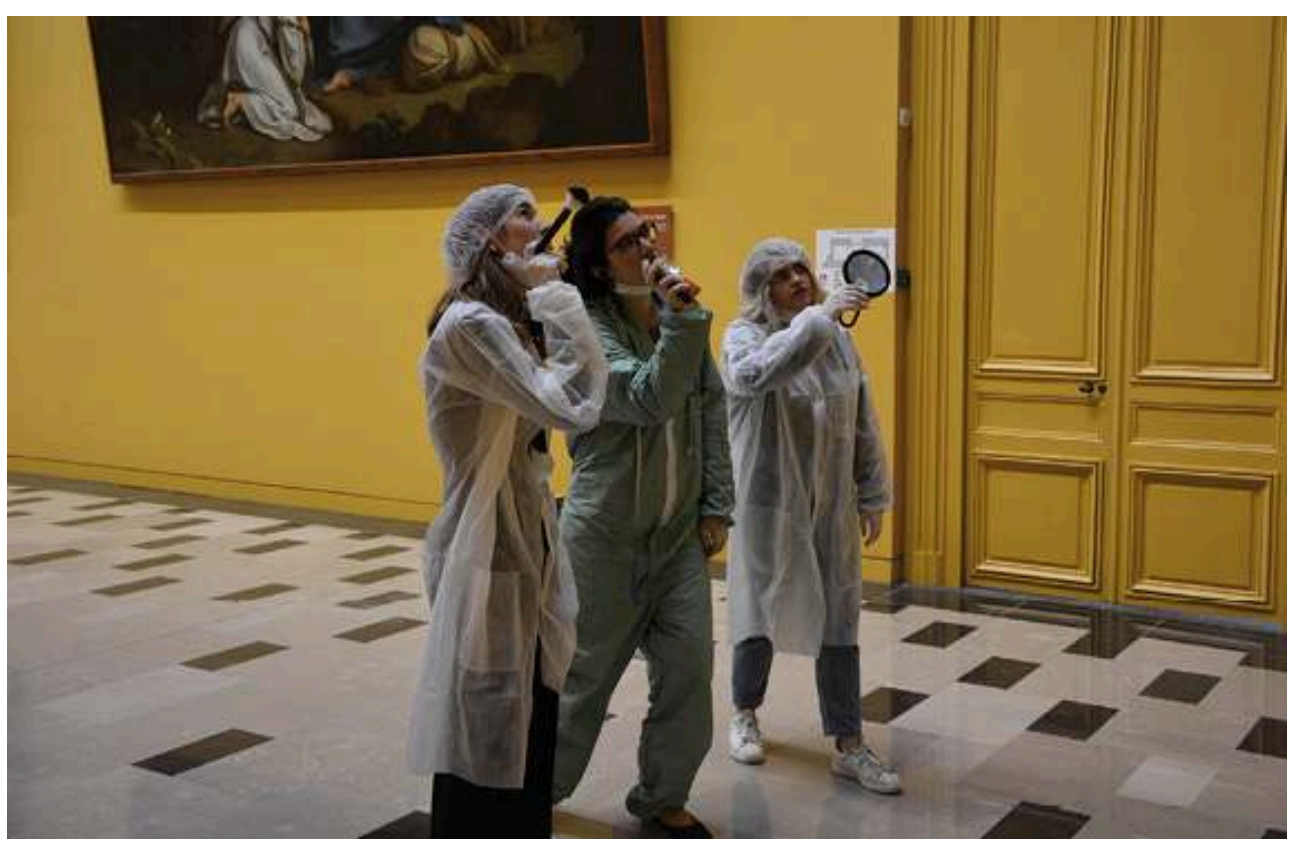

(c) Art de Muser

\section{Déployer des compétences par l'expérience}

7 Si disposer d'un cadre conceptuel et théorique est certes important, l'apprentissage est avant tout une démarche de formation professionnelle et les sessions en CFA sont essentielles pour acquérir des outils méthodologiques et pour intégrer des techniques au travers de journées de spécialisation. Ainsi la formation doit compléter l'expérience 
en entreprise, pour offrir une complémentarité cohérente, dotant l'apprenti des ressorts pour accomplir ses missions, mais aussi pour réussir son insertion professionnelle. Si le CFA dispense des connaissances, c'est leur mise en œuvre sur le terrain qui permet de les transformer en compétences. Le CFA est un lieu où les exercices et les mises en pratique sont multiples, ce qui vient compléter les missions conduites en entreprise. Pour cela, la formation n'est pas semblable à un cursus universitaire classique, car il importe que les formateurs soient proches des logiques de l'entreprise et que les travaux conduits au CFA lors des sessions de regroupement préparent pleinement à la vie professionnelle.

\section{Recourir à une conception pédagogique spécifique}

8 Ainsi, les formateurs doivent disposer non seulement d'une réelle implication dans le champ professionnel pour en connaître les ressorts, d'une reconnaissance par le milieu, mais aussi disposer d'une vision sur le secteur. Former de futurs professionnel des musées ou des expositions exige de disposer d'une conception affirmée de l'avenir du secteur mais aussi de la lier à une démarche pédagogique professionnalisante. Le master Muséographie Expographie (MEM) de l'université d'Artois développe ainsi une démarche de pédagogie active qui consiste à placer l'apprenant au centre du dispositif en lui confiant la réalisation de missions issues de commandes institutionnelles. Aucun travail fictif n'est réalisé et les cours ont été remplacés par des sessions de formations ouvertes, des réalisations in situ, comme des séances en ateliers ou des workshops et par des exercices conduits sur le terrain. La formation se déroule en itinérance dans les sites et les lieux d'exercice des apprentis ou dans des sites aux problématiques semblables à ce qu'ils connaîtront dans leur vie professionnelle. Le master a reçu le label SUP'Artois pour la cohérence de sa pédagogie active et est classé $8^{\mathrm{e}}$ meilleure formation management culture en France par SMBG pour la $5^{\mathrm{e}}$ année consécutive.

\section{Développer le partenariat entre le lieu de formation et l'entreprise}

9 Évidemment, cela suppose de nouer des relations étroites entre le CFA et les entreprises du secteur, qui en muséographie peuvent être : des musées, des centres d'art, des centres d'interprétation, des centres de sciences, des parcs naturels, des mémoriaux, des entreprises privées, des fondations, des agences et d'autres prestataires des lieux d'exposition. Le CFA peut ainsi proposer à l'entreprise de conduire des chantiers communs. Un terrain d'exercice pédagogique tout-à-fait profitable à toute la formation. Il arrive que des relations nouées à l'occasion d'un apprentissage dans une structure donnent lieu ensuite au développement de projets communs. C'est le cas par exemple de journées organisées entre Universcience et le master MEM ou encore de projets avec le Muséum national d'Histoire naturelle, le musée de La Chartreuse de Douai, le musée d'Histoire naturelle de Lille, le Forum départemental des Sciences... 
Chaque année, une commande de collecte ethnographique est assurée par la promotion de MEM pour le compte d'un commanditaire : auprès des marins pécheurs d'Étaples pour le Conseil départemental, auprès des anciens mineurs pour Mineurs du Monde et de la communauté polonaise pour l'ICEP... À ce jour, six collectes ont été conduites.

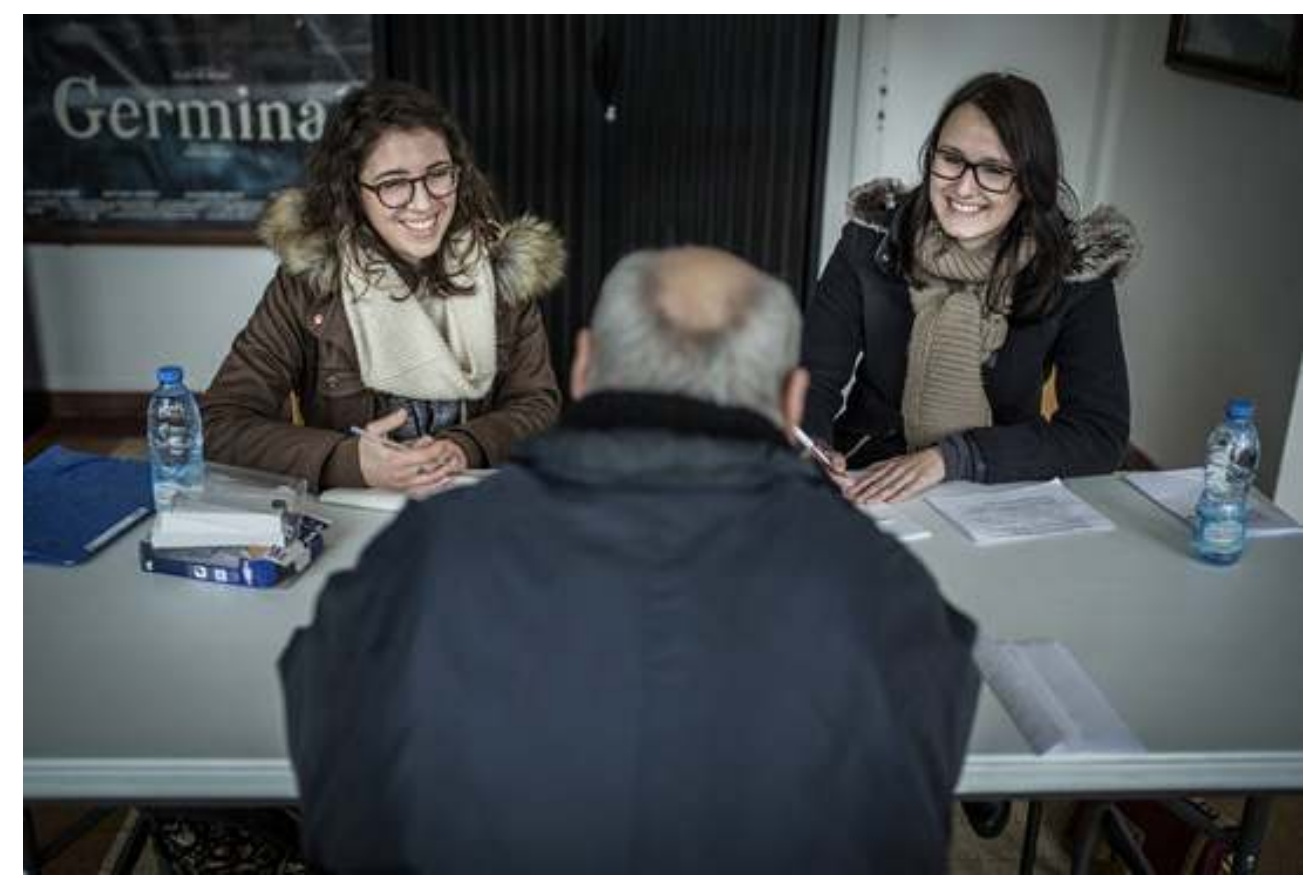

(c) Alessio Vecchié

\section{Les intérêts de l'apprentissage pour les collectivités publiques}

Disposer d'un apprenti, c'est d'abord contribuer à la formation des jeunes professionnels et futurs collègues. C'est avoir une exigence de transmission, mais c'est aussi disposer d'un collègue salarié pour une année entière dont le niveau de qualification, la motivation, et l'encadrement sont sans équivalent. Il n'est pas sans importance que le coût salarial soit moins élevé qu'avec un salarié classique, de l'ordre de $61 \%$ pour un master 2 (variable à la marge selon la structure, son statut, son nombre de salariés...).

11 Avec la RGPP (Révision Générale des Politiques Publiques), les établissements dépendant de la fonction publique sont conduits à ne pas remplacer un fonctionnaire sur deux, phénomène sans doute amplifié à l'avenir par la baisse de dotations des collectivités territoriales, et l'érosion des subventions. Or, si un apprenti est un véritable salarié, en revanche son recrutement ne compte pas dans la masse salariale. Il s'agit là d'un argument de plus en faveur de l'apprentissage.

12 Si le recrutement de l'apprenti a lieu de septembre à septembre pour se caler avec le rythme de la formation, il est possible pour l'entreprise de faire commencer le contrat 3 mois avant, et de le poursuivre 3 mois après la fin de formation. 


\section{Les conditions nécessaires à l'accueil d'un apprenti}

Seules les entreprises privées, et donc aussi les associations, versent une côte part pour la taxe d'apprentissage sur les charges salariales pour alimenter le fond de financement de l'apprentissage. Celles d'entre-elles qui recrutent un apprenti peuvent ainsi déduire le coût de formation de ce versement obligatoire. Les collectivités publiques peuvent également recruter des apprentis, à condition de s'acquitter d'un coût de formation puisqu'elles ne cotisent pas par ailleurs. Celui-ci est dépendant de la formation, du nombre d'apprentis, de l'équilibre budgétaire atteint. Rappelons que légalement les formations en apprentissage ne sont pas censées mixer les publics, ceux de la formation initiale et ceux de l'apprentissage. Vérifier que tous sont bien en apprentissage est aussi un gage de qualité de la formation choisie. L'État ne versant pas de dotations pour les apprentis, l'équilibre financier doit s'établir avec les organismes dédiés. Les promotions sont réduites, condition indispensable pour un suivi sérieux et personnalisé de chacun des apprentis.

Une évaluation de l'opération L'Entreprise à l'œuvre, chez Renault-Flins, commande du ministère de la Culture et de la Communication. Chaque année un travail avec une institution est conduit : inventaire d'une collection, diagnostic-préconisation, évaluation d'exposition ou d'un multimédia...

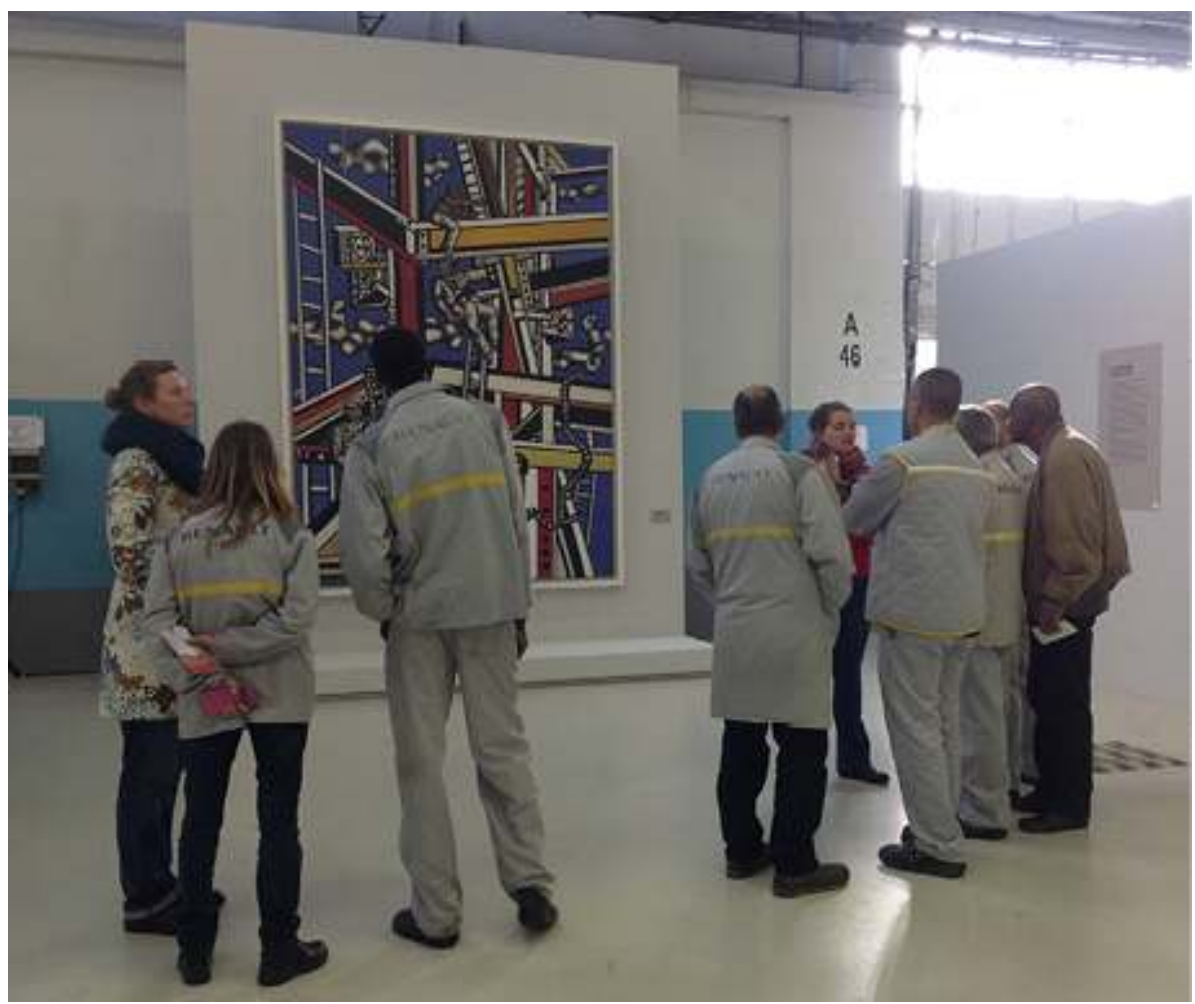

(c) Art de Muser

\section{Les conditions de mise en place d'une formation par apprentissage}

Non seulement le CFA doit disposer d'un encadrement formé et conscient des enjeux que suppose une formation réellement professionnelle (bien éloignée d'une mosaïque 
de cours agrémentée d'un stage), mais l'exigence veut que la promotion soit entièrement composée d'apprentis, pour conduire un accompagnement au même rythme et répondre aux exigences de l'alternance. Pour être agréé comme CFA, le porteur de projet doit présenter un dossier conséquent, dans lequel figure l'appui du secteur professionnel, puis défendre devant les commissions paritaires la rigueur, la cohérence et la viabilité de son projet. Dans le secteur culturel, il y a très peu d'élus car la viabilité économique est loin d'être évidente.

\section{L'exemple du master Expographie Muséographie de l'université d'Artois}

Parce qu'il a été conçu dés le départ dans la logique de l'alternance et avec une forte implication professionnelle, le passage à l'apprentissage a pu se faire rapidement. Dès la première année, 15 apprenties, puis 17 apprenties en seconde année, se sont réparties dans toute la France dans des institutions et des entreprises diverses : musées municipaux, collectivités territoriales (mairie, département...), associations, agences, entreprises, établissements publiques, SEM... La réussite tient à la confiance accordée par le secteur professionnel envers un master sans équivalent par son ancrage au quotidien dans la vie professionnelle, mais aussi par la qualité de son recrutement, les jeunes professionnels donnant pleine satisfaction une fois recrutés. Au terme de chacune des deux premières promotions, trois apprenties ont été recrutées en CDI par l'entreprise, et plusieurs ont vu leur contrat prolongé en CDD.

Le master forme dans quatre domaines : la muséographie, conception d'exposition ; la conservation et régie ; la médiation et l'action culturelle ; l'administration et coordination de projets. Volontairement transversal, il forme pour travailler dans les arts, les sciences, l'ethnographie, l'histoire ou encore dans les disciplines artistiques. Ce qui compte c'est l'accompagnement du projet de chacun, au plus près de ses compétences et de ses espérances.

\section{La responsabilité de l'entreprise}

En transmettant son savoir-faire, l'entreprise s'engage évidemment à proposer des missions pour que le jeune collègue puisse déployer ses compétences et dispose de conditions épanouissantes. Comme cela devrait être fait lors du recrutement d'un stagiaire, l'entreprise devrait vérifier l'adéquation entre le programme de formation et les missions confiées, le sérieux de la formation et de son encadrement ${ }^{1}$. L'apprentissage n'est pas simplement une main d'œuvre bon marché, le dispositif prend tout son sens quand une réelle complémentarité existe, mieux un partenariat, voire une complicité pour accompagner au mieux l'encadrement partagé. Ceci suppose que l'entreprise s'intéresse au lieu de formation et à ses activités, à l'image de l'intérêt des formateurs pour les actions de l'entreprise. L'avenir de l'apprenti est d'intégrer le monde professionnel, aussi l'éthique interdit de remplacer un apprenti par un autre sur de mêmes missions. Enfin l'opération est souvent pleinement satisfaisante quand l'entreprise sait donner toute sa place à l'enthousiasme de l'apprenti en lui confiant des responsabilités. Mieux en décidant de le garder en l'embauchant au terme de son apprentissage. 
Des séminaires, des colloques, des journées d'étude, visent à mixer les jeunes en formation et les professionnels sur un sujet donné. Par exemples sur les mémoires d'exposition au Palais des Beaux-Arts de Lille ou sur les collectes participatives au musée In Flanders Fields à Ypres.

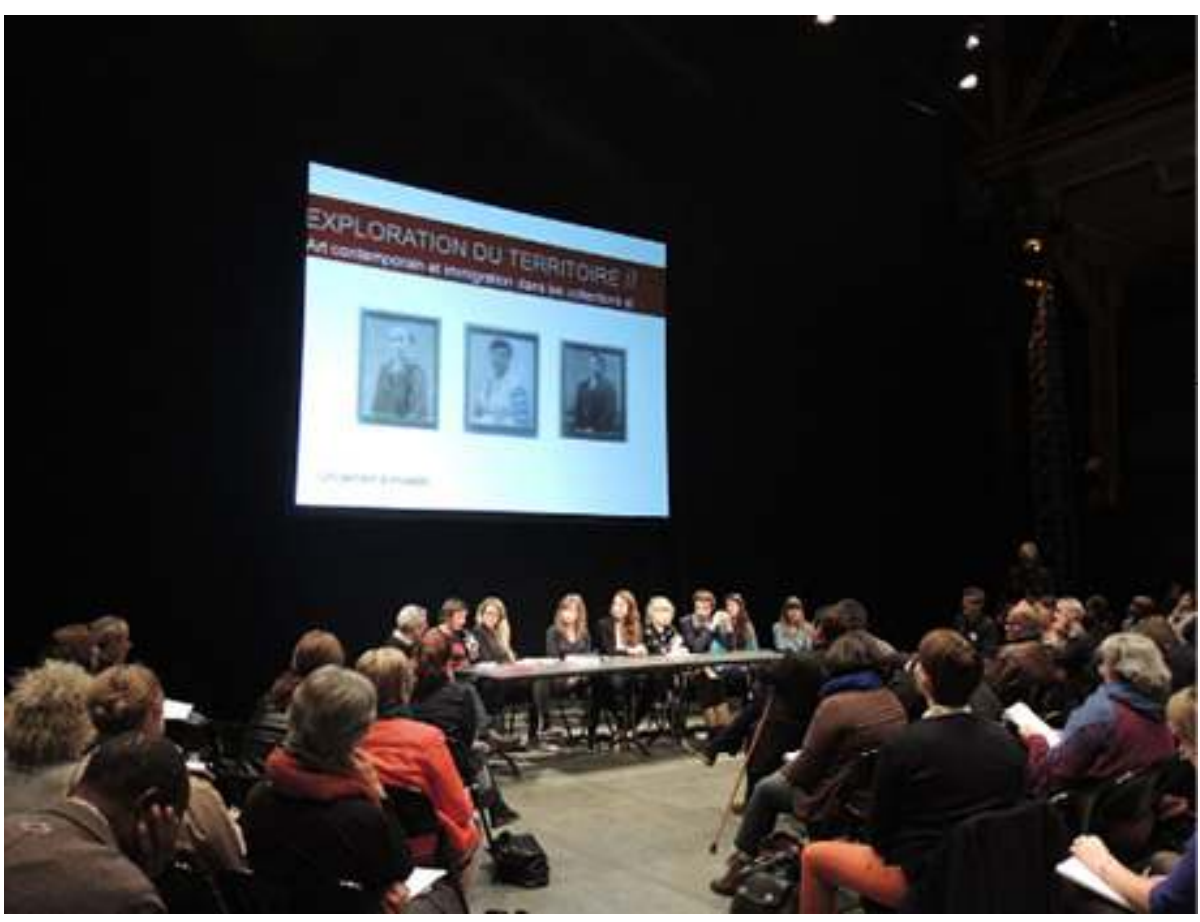

(C) Art de Muser

\section{Les modalités du recrutement d'un apprenti}

Il faut tout d'abord s'assurer de l'accord de son autorité de tutelle et des fonds disponibles pour ce faire. Un échange entre le CFA et l'entreprise en amont est souhaitable pour bien calibrer la démarche. Ensuite établir une fiche descriptive de poste, et la faire parvenir idéalement en mai de chaque année. Une bourse des offres et des demandes peut ainsi être conduite pour chercher la meilleure adéquation entre les propositions et les profils des futurs apprentis. Ensuite, après accord des parties entre elles, les procédures administratives sont simples avec l'établissement d'un contrat de travail qui peut être entériné rapidement par les parties concernées (entreprise, apprenti et CFA).

\section{Conclusion}

17 À l'heure des moyens contraints que connaissent les structures mais en même temps de l'exigence accrue de professionnalisation, l'apprentissage représente une voie intéressante pour tous. C'est pour l'entreprise un moyen de tester une future recrue pendant un an, de le former à "l'esprit maison", de participer à l'effort collectif de transmission et de formation des futurs collègues. S'il est illusoire de croire que tous les apprentis seront recrutés au terme de leur apprentissage, c'est malgré tout une expérience incomparable dans un curriculum vitae. Pour les formateurs, c'est un moyen de rester en contact quotidien avec le milieu professionnel. Car une véritable formation 
professionnelle, ce n'est pas faire des cours théoriques ou méthodologiques d'un côté et accoler une immersion en entreprise de l'autre, c'est de savoir marier au quotidien les allers-retours, de multiplier les expériences concrètes de mises en situation, variables et différentes, au CFA comme en entreprise. L'apprentissage est une proposition nouvelle dans le champ culturel, aux musées et centres d'exposition de s'en saisir pour inventer de nouvelles solutions pour les métiers du musée.

Toutes les informations sur l'apprentissage dans l'onglet sur notre plateforme, bouquet de liens du master MEM : http://www.pearltrees.com/schaum

Site Internet de la formation:

http://formation-exposition-musee.fr

Site internet L'Art de Muser, association de filière:

http://lartdemuser.blogspot.fr

21 Facebook L'Art de Muser :

https://www.facebook.com/Lart-de-muser-Association-du-Master-Expo-

Muséographie-325462064147699/

www.lapprenti.com/

\section{NOTES}

1. Chaumier, S. Réflexions sur les stages et les conditions d'accueil d'un stagiaire dans une institution culturelle, La Lettre de l'Ocim, n' ${ }^{\circ}$ 152, mars-avril 2014, pp. 5-13.

\section{RÉSUMÉS}

Depuis deux ans, la formation par l'apprentissage est devenue possible pour les métiers de la conception d'exposition, de la médiation et plus généralement de l'administration de projets culturels : l'auteur expose les conditions de mise en place de ce type de formation et met en lumière les avantages de cette approche professionnelle tant pour l'apprenti que pour les structures d'accueil publiques et privées.

\section{INDEX}

Mots-clés : Exposition, muséographie, apprentissage 
AUTEUR

SERGE CHAUMIER

Responsable du Master Expographie Muséographie, université d'Artois.

serge.chaumier@univ-artois.fr 